\title{
PER UN'INTERPRETAZIONE COMUNICATIVA DELLA PUNTEGGIATURA NELLE GRAMMATICHE FRANCESI DEL SECOLO XVIII: ESEMPI DI LETTURA MANZONIANA
}

\author{
Arianna Redaelli ${ }^{1}$
}

Oggetto del breve studio che segue sono i capitoli dedicati all'interpunzione in alcune tra le principali grammatiche francesi del secolo decimottavo, e in particolare nella Grammaire françoise sur un plan nouveau (1709) di Claude Buffier, nei V rais principes de la langue française (1747) dell'abate Girard e nella celebre Grammaire générale (1767) di Nicolas Beauzée, preceduta dalla compilazione dell'articolo Ponctuation (1751) per l'Encyclopédie di Diderot e D'Alembert ${ }^{2}$. L'intento è quello di segnalarne la modernità e la profondità d'analisi, che le differenziano dalle coeve italiane, e l'ascendente che esse esercitarono sulla sintassi moderna, non solo francese ${ }^{3}$. Di qui la decisione di riportare, in conclusione, alcuni esempi manzoniani, tratti dai Promessi Sposi ${ }^{4}$, che gettano luce sulla presenza di diversi punti di contatto tra l'opera e le considerazioni interpuntive svolte in ambiente d'oltralpe.

\section{Punteggiatura E GRAMmatiche: IL SETTECENTO itALiANO}

L'arte del puntare, la cui storia recente è strettamente connessa allinvenzione della stampa a caratteri mobili, fatica, in Italia, a trovare posizione stabile ed estesa nei supporti

\footnotetext{
${ }^{1}$ Università degli Studi di Milano. Questo articolo è tratto dalla tesi di Laurea Magistrale in Lettere Moderne dal titolo Tra Ventisettana e Quarantana: il ripensamento del sistema punteggiatura nei Promessi Sposi. Primi sondaggi e inquadramento del contesto (relatore: prof. Giuseppe Polimeni; correlatori: prof. Massimo Prada - prof.ssa Monica Barsi), discussa nell'a.a. 2019/2020 presso l’Università Statale di Milano.

${ }^{2}$ Non si dimentichino, d'altra parte, opere di grammatici altrettanto centrali, quali Restaut e Du Marsais, che trovano posto in nota. L'esigenza di selezionare gli autori di cui trattare in corpo di testo è dettata da esigenze di comprensione, non meno che di attuabilità dello studio.

${ }^{3}$ Così, in proposito, già Nina Catach (1996: 35): «Dès le début du XVIII e s., Buffier (1709), Grimarest (1707), Girard et autres grammairiens fondateurs de la Grammaire générale se penchent sur le problème de la ponctuation. On pourrait presque dire que c'est à travers elle qu'ils abordent les problèmes de la syntaxe moderne, et qu'ils fondent ainsi en même temps une science de l'écrit, comprise comme partie pleinement reconnue de la langue». E Annette Lorenceau, in un lavoro sull'argomento (1998: 363-364) «A vrais dire, les grammairiens des $19^{\text {ème }}$ et $20^{\text {ème }}$ siècles ont fait croire qu'il existait une ponctuation "moderne" qu'il fallait utiliser, mais en réalité ils se sont contentés d'essayer d'établir des règles, d'ailleurs très confuses, pour leurs contemporaines [...]. C'était renier des prédécesseurs de valeur: les grammairiens du $18^{\mathrm{ème}}$ siècle. Les grammairiens-philosophes ont en effet réservé une place importante dans leurs écrits à la ponctuation; ils ont essayé de la replacer dans l'ensemble de la langue et d'exposer un véritable système de ce que Beauzée appelle "la partie essentielle de l'orthographe"».

${ }^{4}$ Il romanzo manzoniano accoglie usi interpuntivi eterogenei (moderni, arcaici, sintatticamente coerenti o idiosincrasici), che tuttavia attestano la peculiare attenzione dell'autore nei confronti del tema e l'intensità delle sue riflessioni, spesso coincidenti con quelle, teoriche, di origine francese. A tutti i grammatici illuministi citati, e al Beauzée soprattutto, Manzoni fa infatti puntuale riferimento nel saggio inedito e incompiuto Della lingua italiana, dimostrando una conoscenza approfondita delle loro opere, non meno che una vicinanza d'intenti tale da potersi quantomeno tradurre in stimolo, per l'autore dei Promessi Sposi, alla modernizzazione della propria punteggiatura in direzione comunicativo-testuale.
} 
linguistici di riferimento, almeno sino a Ottocento inoltrato. Le grammatiche settecentesche, infatti, pur documentando un interesse crescente nei confronti del tema, nondimeno vi riservano poche pagine, di solito accomodate a concludere la trattazione, e forniscono pratiche e sommarie regole d'uso oscillanti tra un'interpretazione pausativa e un'interpretazione sintattica dei segni ${ }^{5}$, e corredate da inevitabili eccezioni. Rinunciando a un'analisi metodica e speculativa e in accordo con le indifferibili esigenze della prassi, si fanno così ricetto delle abitudini più diffuse, e accordano largo spazio all'arbitrio e al gusto personale dei singoli scriventi: aspetti, questi ultimi, a lungo ritenuti congeniti del sistema. Anche il repertorio dei segni - che include solitamente il punto, la virgola, i due punti e il punto e virgola, e solo a volte gli espressivi - rimane a lungo immutato ${ }^{6}$.

Il Corticelli, per citare un esempio illustre, riserva alla punteggiatura solo cinque pagine delle sue Regole ed osservazioni della lingua toscana, avanzando una proposta di tipo misto: se i segni interpuntivi sono stati «inventati da' Gramatici per contrassegnar le fermate, o sieno pause del parlare» ${ }^{7}$, la controparte sintattica viene avvertita laddove egli, preoccupato «di definire il valore (relativo) della pausa indicata e la posizione del segno interpuntivo nell'ambito del periodo» ${ }^{8}$, riferisce le caratteristiche costitutive individuali dei simboli considerati. Seguono, in ordine, un elenco di norme esatte (e coerenti con il fine didattico dell'opera), una lista d'esempi d'autore, e cinque osservazioni di stampo grammaticale sui vari usi della virgola.

Ancor più deludente ${ }^{9}$, dal punto di vista interpuntivo, è la Gramatica ragionata della lingua italiana di Francesco Soave, pubblicata, dapprima in forma adespota, nel 1771. L'impressione di marginalizzazione dell'argomento è testimoniata, di nuovo, non soltanto dall'esiguità del materiale, dacché la tematica è risolta in poco più di una pagina, ma anche dalla sua ubicazione: il capitolo, accluso alla quinta parte del testo - quella finale, dedicata all'ortografia - è l'ultimo dell'intera trattazione. A una concisa definizione introduttiva, che sembrerebbe rivelare il ruolo prevalentemente prosodico attribuito alla punteggiatura $^{10}$, è fatto qui seguire un rapido riepilogo dei segni, nel quale la terminologia sintattica sovrasta quella riferita all'oralità. A risolvere le problematiche intorno alla collocazione della virgola, infine, l'abituale concessione: «Ognuno può in questo seguir l'uso, che più gli piace, e noi pure ci siamo serviti or dell'uno, or dell'altro modo, secondo che ci è sembrato tornar più comodo» (Soave, 1771: 303) ${ }^{11}$.

\section{Da BufFier a BEAUZÉE: LA PUNTEgGiatura NELLE GRAMMATICHE ILLUMiNiSTE FRANCESI}

Simili osservazioni e tanta confusione non possono essere estese all'ambiente illuminista francese, pure così vicino, geograficamente e linguisticamente, all'Italia. Anche

\footnotetext{
${ }^{5}$ La distinzione tra $\mathrm{i}$ due modi di intendere l'interpunzione, d'altra parte, non è in quest'epoca sempre esplicita, intenzionale od opportuna, essendo sprovvista di un saldo pensiero teorico che la sorregga.

${ }^{6}$ Per una trattazione completa e dettagliata sull'argomento, si veda Fornara (2008: 159-177).

${ }^{7}$ Corticelli (1745: 462).

${ }^{8}$ Persiani (1998: 134).

${ }_{9}^{9}$ Deludente anzitutto perché il lavoro, sebbene si situi «nella linea che congiunge (e non divide) il pensiero grammaticale di Port-Royal all'Encyclopédie» e cerchi «una via mediana tra atteggiamento empirico e atteggiamento speculativo, in armonia con il percorso seguito dagli enciclopedisti» (Fornara, 2001: 18-19), si pone, nei riguardi del tema, in modo decisamente meno attento e costruttivo dei suoi antesignani.

10 «L'uso dei punti, e delle virgole si è introdotto per indicare le pause del discorso, e distinguerne i sensi» (Soave, 1771: 302).

11 Affini, infatti, le cautele di altri grammatici contemporanei al Soave. Tra questi, Benedetto Rogacci (1711: 355), che definisce l'interpunzione «materia sì minuta, e sì dipendente dall'arbitrio», e Pier Domenico Soresi (1756: 87), che rinviene la soluzione finale delle questioni relative all'interpungere nel «discreto giudizio» del giusto mezzo tra la scarsità e l'abbondanza dei segni.
} 
in Francia la punteggiatura è genericamente considerata come «un sistema di segnali che da una parte indicano visivamente pause, attese e riprese della respirazione per la lettura ad alta voce, e dall'altra rivelano l'organizzazione del senso distribuito nei diversi costrutti linguistici» (Barsi, 2008a: 275); ma l'argomento - alimentato dall'attribuzione di una certa autonomia al codice dello scritto, progressivamente emancipatosi dall'oralità - conosce declinazioni differenti all'interno delle varie opere, e dà vita a nutriti dibattiti di respiro ampio e interdisciplinare, la cui eredità grammaticale, sebbene nel suo minuto evolversi sia perlopiù nota ai soli specialisti, giunge sino a oggi.

La concessione di uno spazio consistente al tema è pressoché comune alla totalità dei lavori e si affianca all'individuazione di repertori inclusivi e puntuali; consueti anche $\mathrm{i}$ riferimenti all'irregolarità e alle anomalie di un sistema a lungo trascurato dalle grammatiche e dai supporti linguistici di riferimento, e alle libertà stilistiche dei singoli autori. L'enunciazione dei segni, soprattutto, è supportata da osservazioni e sforzi d'esegesi che, lungi dal limitarsi all'opposizione dicotomica tra pausazione e sintassi, anticipano di qualche decennio gli interventi di alcuni grammatici italiani del tardo Ottocento $^{12}$, nonché dei moderni studiosi d'interpunzione ${ }^{13}$, sul valore comunicativotestuale della punteggiatura ${ }^{14}$.

La distanza tra le grammatiche italiane e quelle francesi si consuma sin dalle considerazioni esibite in sede preliminare. Se il filosofo, pedagogo, storico e autore di punta della scuola gesuitica, Claude Buffier, nella Grammaire françoise sur un plan nouveau, si limita a definire la punteggiatura come «la maniére d'employer divers signes, pour distinguer diférentes parties du discours» ${ }^{15}$, riconosce però sin da subito l'effettivo valore dei segni nel complesso della testualità ${ }^{16}$ e condanna - e non avalla - l'incontrollata atomizzazione del sistema, di volta in volta flesso in relazione alle preferenze e alle personali valutazioni degli scriventi. Constata, quindi,

qu'il est tres dificile ou même impossible de faire sur la ponctuation un sistême juste, \& dont tout le monde conviéne; soit à cause de la varieté infinie qui se rencontre dans la maniére dont les phrases \& les mots peuvent être arangez; soit à cause des idées diférentes que chacun se forme à cette ocasion (ivi: 420).

\footnotetext{
${ }^{12} \mathrm{Ci}$ si riferisce, in particolare, al pregevole lavoro di Giovanni Gherardini, la Lessigrafía italiana, dato per la prima volta alle stampe nel 1842 e perlopiù ignorato sino alla fine del secolo; alla Sintassi italiana dell'uso moderno di Raffaello Fornaciari (1881); e ad alcune grammatiche e trattatelli d'impronta manzoniana, quali, rispettivamente, la Grammatica italiana di Giulio Cappuccini e Luigi Morandi (1894) e La punteggiatura nel periodo italiano e il suo uso di Camillo Gastaldi (1895).

$13 \mathrm{Il}$ rimando è agli studi del gruppo di Basilea. Per nozioni più precise, cfr. almeno Ferrari et al. (2018).

${ }^{14}$ Secondo tale concezione, essa compie, a seconda dei segni e dei casi considerati, due ordini di operazioni: da un lato, la segmentazione del testo «nelle sue unità semantico-pragmatiche, concorrendo a volte alla loro gerarchizzazione»; dall'altro, l'introduzione di «valori semantici interattivi come ad esempio le implicature e i valori illocutivi» (Ferrari, 2017: 152-153). In tale quadro, le occorrenze prosodiche e sintattiche che talvolta intervengono nello scritto sono valutate come epifenomeni, ossia come fenomeni più o meno duraturi attivati dalla ratio interpuntoria, che è di natura, appunto, comunicativa.

15 Buffier (1709: 418). All'argomento l'autore dedica una quindicina di pagine, che chiudono la sezione intitolata Des diverses figures qu'admet l'Ortographe Françoise autres que les caractéres de letres, \& qui sont communes à l'anciéne \& à la nouvelle Ortographe.

16 «Cette pratique introduite en ces derniers siécles dans la Grammaire est des plus avantageuses; elle prévient diverses ambiguitez qu'on peut rencontrer à l'ocasion d'un mot qui se raporteroit à une phrase ou à une période, plutôt qu'à une autre. C'est par une omission de points \& de virgules bien marquées, qu'il s'est trouvé des dificultez insurmontables, soit dans le texte de l'Ecriture-Sainte, soit dans l'exposition des Dogmes de la Religion, soit dans l'énonciation des Loix, des Arrêts \& des Contrats de la plus grande conséquence pour la vie civile» (Buffier, 1709: 419).
} 
La posizione di Buffier è fortemente critica. Dopo una breve rassegna dei caratteri ${ }^{17}$, classificati secondo percezione comune - vale a dire in relazione al raggiungimento, totale o parziale, del senso, alla completezza della frase e alla complementarità d'impiego propria dei segni stessi -, lo studioso torna a lamentare la statica opacità di tali proposte, per nulla supportate da una riflessione ricca e coesa e da riscontri testuali condivisi ${ }^{18}$, prefiggendosi perciò di offrire ai lettori alcuni dettagli espositivi (non rigide prescrizioni) che possano facilitare un impiego più corretto e consapevole dei simboli d'interpunzione.

Ancora più sensibile alla stretta interrelazione tra scritto e parlato, il cui incontro nel testo è siglato dalla presenza dei segni di punteggiatura, è l'abate Gabriel Girard che, in Les V rais principes de la langue française, ou La parole réduite en méthode conformément aux lois de l'usage, en seize discours, così definisce l'art de la ponctuation:

Puisqu'il se fait entre la main \& les organes de la prononciation un commerce réciproque pour la manifestation de la pensèe, l'une peignant ce que l'oreille entend, \& les autres faisant entendre ce que l'euil voit; il faut une exactitude entiere dans ce commerce; afin que la Parole écrite réponde parfaitement aux modifications \& à toutes les circonstances de la Parole prononcée, dont elle est l'image. Or comme la prononciation exige des repos, pour faire de nouvelles provisions d'air à mesure qu'elle en dépense; cela est cause que la voix ne marche pas desuite, mais par intervales mesurés sur le sens \& sur la nature de la frase. Ainsi l'Ecriture doit présenter aux yeux ces repos \& ces intervales aussi bien que les sons \& les articulations. Elle s'en aquite par le moyen de six caracteres établis à cet usage, savoir, le POINT, les DEUX POINTS, la VIRGULE PONCTUE'E, la SIMPLE VIRGULE, le POINT INTERROGANT, \& le POINT EXCLAMATIF autrement dit POINT D'ADMIRATION.

C'est à savoir placer chacun de ces caracteres que consiste l'art de la PONCTUATION ${ }^{19}$.

Pure trattenuto nel valutare la cardinalità dei segni interpuntivi, il grammatico ne riconosce però il ruolo attivo nel sistema linguistico e ne individua ed esalta i pregi, tra $i$ quali egli annovera il fondamentale contributo a un'adeguata intelligenza del testo:

Il est très vrai que par raport à la pureté du langage, à la netteté de la frase, à la beauté de l'expression, à la délicatesse \& à la sòlidité des pensées la Ponctuation n'est que d'un mince mérite [...]. Mais je crois qu'il n'est point indigne de l'auteur le plus célebre d'y donner quelque attention; lorsqu'après avoir fait \& poli son ouvrage, il le copie pour l'impression. [...] La Ponctuation soulage \& conduit le Lecteur. [...] Elle contribue à l'honneur de l'Intelligence [...]. Elle tient en regle l'attention de ceux qui écoutent, \& leur fixe les bornes du sens. Elle remédie aux obscurités qui viennent du stile. Tels sont ses effets ${ }^{20}$.

\footnotetext{
${ }^{17}$ Si tratta di punto, virgola, punto e virgola, due punti, punto esclamativo e interrogativo. Pur non trovando posto tra i caratteri di punteggiatura, è però compreso nel medesimo capitolo della grammatica di Buffier anche il trattino, «une petite ligne comme celle-ci (-)» (ivi: 412).

18 «Voilà ce qu'on dit ordinairement, \& ce qui ne donne pas des idées fort précises: car qu'est-ce que distinguer des noms, des verbes, des parties d'une période, qui ne sont pas jointes nécessairement? Qu'estce qu'une période compléte \& un sens entiérement achevé? Qu'est-ce qu'un sens moins complet ou plus achevé?» (ivi: 420). L'osservazione è tanto più acuta se si considera il fatto che venga sollevata agli albori di un'epoca in cui «le nombre des auditeurs est infiniment plus élevé que le nombre des lecteurs», e il ruolo della punteggiatura dovrebbe essere quello di «faciliter la lecture à haute voix» (Lorenceau, 1998: 365).

${ }^{19}$ Girard (1747: 433-434). L'intervento sull'interpunzione occupa, nell'opera, una trentina di pagine.

${ }^{20}$ Ivi: 435.
} 
La modernità dell'opera, tuttavia, sta nel criterio che governa la scelta e l'esatta collocazione dei simboli interpuntivi, che è dichiaratamente comunicativo: pertiene infatti al grado di separazione o, viceversa, di connessione dei significati. L'autore ne individua quattro, gerarchicamente ordinati. Il primo è quello dei sens constructifs, ed è caratterizzato da significati tra loro distinti ma uniti da un'unica costruzione frasale; il secondo grado è rappresentato, invece, dalla circostanza di frasi altresì distinte, le quali concorrono tuttavia ad un unico senso complessivo, ed è quello dei sens relatifs; il terzo, dei sens partiels, ricalca le condizioni del precedente, con la sola differenza che, in quest'ultimo caso, le frasi sono in maggior misura autonome. Al quarto grado, quello dei sens intégraux, appartengono infine contenuti isolati, il cui unico legame è composto da «l'analogie des pensées \& la convenance du sujet $\rangle^{21}$ : essi possono essere semplici o complessi - vale a dire, compresi in un unico periodo oppure dipendenti da più di uno - e possono influenzare, alternandosi e combinandosi, anche la qualità stilistica del testo ${ }^{22}$. Affinché tali principi possano essere applicati, Girard si richiama rapidamente a quanto dichiarato sulla frase nel terzo dei suoi discorsi: essa è, «par le concours des mots qui la composent, la représentation d'une pensée: \& ce concours est ce qu'on nomme RÈGIME» ${ }^{23}$; il numero dei membri e delle rispettive funzioni che la compongono giunge invece sino a sette («Subjectif, Attributif, Objectif, Terminatif, Circonstanciel, Conjonctif, \& Adjonctif $\left.\rangle^{24}\right)$.

All'introduzione dei concetti elencati, segue il commento della loro applicazione in relazione ai quattro segni principali ${ }^{25}$, di nuovo condotta per gradi progressivi d'indipendenza. In merito ai sens partiels, per esempio, Girard afferma che questi ultimi si distinguono tra loro mediante l'impiego dei due punti; in termini di stampa, tale distinzione è definita comma, voce greca che significa appunto 'sezione' o 'frammento', poiché si occupa di scomporre il periodo in parti. Per rendere più marcata e comprensibile la differenza tra i due punti e il punto e virgola, l'autore propone poi il confronto tra due brevi testi, simili per argomento ma sintatticamente divergenti ${ }^{26}$; precisa però che talora, in particolare nel caso dell'enumerazione di significati autonomi gli uni rispetto agli altri ma tutti subalterni a una medesima proposizione, i primi, ossia i due punti, possono trovarsi a distinguere non soltanto i membri principali di un periodo, bensì anche le subordinate, funzione analoga e sovrapponibile a quella generalmente rivestita dal punto e virgola ${ }^{27}$.

Avendo inoltre ben individuato la capacità, propria dei caratteri interpuntivi, di gerarchizzare i contenuti che prendono parte al discorso, egli avverte che tra le

${ }^{21}$ Ivi: $437-438$.

22 Ivi: 436-438.

23 Ivi: 439. Sulle conoscenze sintattiche preliminari da verificare prima di intraprendere qualsiasi discorso sull'interpunzione, così anche Pierre Restaut (1730: 291-292), che pure definisce quest'ultima come «la maniere de marquer en écrivant les endroits d'un discours où l'on doit s'arrêter, pour en distinguer les parties, ou pour reprendre haleine»: «D. Que faut-il savoir avant que d'entrer dans l'explication de ces différents caracteres? R. Il faut savoir ce que c'est que Phrase \& Période. Il y a de trois sortes de phrases; savoir, la phrase simple, la phrase composée, \& la phrase complexe. Toute phrase (ou proposition) doit avoir au moins un Sujet \& un Attributs. I corsivi sono dell'autore.

${ }^{24}$ Girard, 1747: 439.

25 Stando a Girard, infatti, gli espressivi non esercitano alcuna influenza sulla scala di divisione sintatticosemantica e sono da scegliere soltanto in occorrenza di tono esclamativo o interrogazione.

26 «Voici un exemple de cette ponctuation: l'amour est une passion de pur caprice: il attribue du mérite à l'objet dont on est touché: il ne fait pour tant pas aimer le mérite: jamais il ne se conduit par reconnoijsance: tout est chez. lui gout ou sensation: rien n'y est lumiere ni vertu. Pour rendre plus sensible la différence qu'il y a entre la distinction que doivent marquer les deux points \& celle à qui la virgule ponctuée est affectée, je vas donner à l'exemple raporté un autre tour [...]: l'amour est une passion de pur caprice; qui attribue du mérite à l'objet aimé; mais qui ne fait pas aimer le mérite; à qui la reconnoissance est in connue; parceque chez. lui tout se raporte à la volupté; \& que rien n'y est lumiere ni ne tend à la vertuı (Girard, 1747: 461-462). I corsivi sono dell'autore.

${ }^{27}$ Questo segno, infatti, è prescritto da Girard per i sens relatifs, insieme alla virgola semplice; ai sens constructifs si accompagna, invece, solo quest'ultima, e agli intégraux il punto fermo. 
subordinate non dovrebbe esserci la stessa forza partitiva che sussiste tra le principali: se, infatti, «cellesci sont distinguées par les deux points», al contrario «cellesla ne doivent l'être que par la simple virgule ou par celle qui est ponctuée» ${ }^{28}$. Tale è

la pratique du bon stile; qu'on observe naturellement dans la construction des périodes; quoique souvent les caracteres de la ponctuation soient assez négligés pour confondre dans l'écriture les gradations du sens \& ôter aux yeux la satisfaction de voir toute l'économie de la Syntaxe ${ }^{29}$.

Il culmine della discussione interpuntiva di ambiente francese, tuttavia, è raggiunto grazie al duplice contributo di Nicolas Beauzée. Mentre nell'articolo enciclopedico intestato alla ponctuation Beauzée si limita a definire la punteggiatura «l'art d'indiquer dans l'écriture, par les signes reçus, la proportion des pauses que l'on doit faire en parlantı ${ }^{30}$, nella Grammaire générale la definizione è integrata nel modo che segue:

La Ponctuation est l'art d'indiquer dans l'écriture, par les signes reçus, la proportion des pauses que l'on doit faire en parlant. [...] Le choix des Ponctuations dépend de la proportion qu'il convient d'établir dans les pauses; \& cette proportion dépend de la combinaison de trois principes fondamentaux: $1^{\circ}$. le besoin de respire; $2^{\circ}$. la distinction des sens partiels qui constituent un discours; $3^{\circ}$. la différence des degrés de subordination qui conviennent à chacun de ces sens partiels dans l'ensemble du discours ${ }^{31}$.

Il risultato è significativo soprattutto perché tende a non isolare, bensì ad accorpare, con consapevolezza e coerenza notevoli, il livello pneumatico (per la maggior parte legato alle esigenze della lettura a voce alta) ${ }^{32}$, quello del senso e quello dell'ordine delle unità all'interno del periodo ${ }^{33}$.

Il capitolo sull'interpunzione - che si trova a chiusura del tomo secondo, dedicato agli Éléments de la Syntax - è introdotto da alcune interessanti considerazioni intorno alla sua genesi e sviluppo. La pressoché totale assenza, all'interno di numerosi manoscritti, di qualsiasi sforzo distintivo tra parole, contenuti e proposizioni indurrebbe anzitutto a desumere che «l'art de la Ponctuation étoit ignoré dans ces temps éloignés»; i principi che ne determinano l'impiego, d'altronde, «sont même aujourdhui si incertains, si peu fixés par l'usage uniforme \& confiant des bons auteurs; qu'au premier aspect on est porté à

${ }^{28}$ Girard (1747: 464).

${ }^{29}$ Ibidem.

${ }^{30}$ Beauzée (1973 [1751]: 15).

31 Beauzée (1767: 577-578).

32 Intorno al rapporto tra oralità e scrittura, si sofferma in quegli anni anche Du Marsais. Trattando dei vari tipi d'accento, per esempio, egli indugia sull'accent oratorie, necessario a esprimere e comunicare i sentimenti di chi parla, e subito dopo su «les intervalles que l'on met dans la prononciation, depuis la fin d'une période jusqu'au commencement de la période qui suit; \& entre une proposition, \& une autre proposition: entre une incise, une parenthèse, une proposition incidente, $\&$ les mots de la proposition principale, dans les quels cette incise, cette parenthèse ou cette proposition incidente sont ensermées». Tutte queste «modifications de la voix, qui sont très sensibles dans l'élocution, sont, ou peuvent être marquées dans l'écriture, par des signes particuliers, que les anciens Grammairiens ont aussi appellés accens» (Du Marsais, 1769: 574). Spontaneo il rimando ai segni di punteggiatura, e in particolare alla loro funzione di riferimento per le modulazioni della voce in corso d'eloquio o di lettura non silenziosa. Lo stesso autore, infatti, conducendo poco più avanti qualche riflessione su antiche testimonianze d'abitudini scrittorie, nota: «Quoi qu'il en soit, il est certain que la manière d'écrire a été sujette à bien des variations, comme tous les autres arts. Ainsi, tout ce que l'on peut conclure de ces manuscrits, où l'on ne voit ni distancé entre les mots, ni accens, ni points, ni virgules, c'est qu'ils ont été écrits ou dans des temps d'ignorance, ou par des copistes peu instruis» (ivi: 576-577).

${ }^{33}$ L'integrazione ricorda, anche nella terminologia, la proposta di Girard, che infatti compare come fonte e modello (talora polemico) di confronto in molti luoghi del testo. 
croire que c'est une invention moderne» ${ }^{34}$. Nondimeno, persino negli scritti più antichi, si trovano precoci testimonianze del bisogno di frazionare il testo, per favorire il quale si istituirono alcuni caratteri adatti allo scopo e perfezionati nel corso del tempo, noti, peraltro, ad autori quali Isidoro di Siviglia, Gerolamo di Stridone, Cicerone e Aristotele ${ }^{35}$. Varie influenze intervenute nella storia della scrittura ne limitano, talvolta frenandola del tutto, la fruizione. D’altra parte, continua Beauzée,

si, nonobstant ces considérations, il y a lieu de s'étonner que les anciens ayent fait si peu d'attention à l'art de ponctuer; il seroit presque scandaleux que, dans un siècle éclairé comme le nôtre \& avec les moyens de communication que nous avons en main, nous négligeassions une partie si importante de la Grammaire \& si nécessaire à la clarté de la parole écrite ${ }^{36}$.

Tacendo o mal collocando i segni di punteggiatura, infatti, si incontrerebbero le medesime difficoltà causate, a un diverso livello di comunicazione linguistica, dall'omissione o dall'uso scorretto delle pause all'interno del discorso orale:

les uns comme les autres servent à déterminer le sens; \& il y a telle suite de mots qui n'auroit, sans le secours des pauses ou des caractères qui les indiquent, qu'une signification incertaine \& équivoque, \& qui pourroit même présenter des sens contradictoires ${ }^{37}$.

Non solo: dopo aver appurato la generalizzata disattenzione dei grammatici nei confronti della materia interpuntiva e la tendenza condivisa a minimizzarne la portata e gli effetti, Beauzée precisa, come anticipato nella definizione, che a regolamentare l'uso dei segni interviene anche la partizione dei significati all'interno della frase e il loro grado di dipendenza e subordinazione reciproche, strettamente combinati al bisogno fisiologico di respirare. La disgiunzione dell'un criterio dagli altri, infatti, porterebbe, da un lato, alla meccanica divisione del periodo in sezioni di egual lunghezza e misura - corrispondenti al tempo necessario per riprendere fiato - con il grave rischio di comprometterne il senso; dall'altro, a un esponenziale aumento della libera discrezione autoriale, demandando la maggior parte delle scelte ai giudizi di valore del singolo, e a equivoci nella pronuncia e nella comprensione del testo ${ }^{38}$.

\footnotetext{
${ }^{34}$ Beauzée (1767: 567).

${ }^{35}$ Qualche pagina più avanti, il grammatico precisa che l'«ancienne Ponctuation n'avoit pas les mêmes signes que la nôtre» (ivi: 575). Auspicando, soprattutto grazie al potere d'influenza della stampa e alla correzione postuma degli antichi volumi, la riduzione a un unico sistema di punteggiatura, quello moderno, constata inoltre che tanti sistemi diversi come quelli che si sono avvicendati nel corso dei secoli «multiplient gratuitement les difficultés \& entraînent des dégoûts», rappresentando forse la «source de l'indifférence où l'on paroît avoir été jusqu'à présent sur la perfection du nôtre». E relativamente a quest'ultimo prosegue: «Il gagneroit sans doute à être le seul, \& il diminueroit le danger des méprises: pourquoi ne prendroit-on pas enfin ce parti avantageux? Nos signes de Ponctuation, comme on va le voir, vent suffire, ou peu s'en faut, à toutes les distinctions possibles» (ivi: 576-577).

36 Ivi: 571.

${ }^{37}$ Ivi: 572.

38 «Cicéron semble uniquement fonder l'art de ponctuer sur les besoins des poumons: Clausulas enim, dit-il (t), atque interpuncta verborum anima interclusio atque angustia spiritus adtulerunt. Mais si l'on n'avoit égard qu'aux besoins de la respiration, le discours devroit se partager en parties à peu près égales: il arriveroit de là que souvent on suspendroit maladroitement un sens qui pourroit par là devenir inintelligible; d'autres fois on réuniroit des sens tout à fait dissemblables ou disparates, ou bien la fin de l'expression d'un sens avec le commencement d'un autre. Tout cela prouve qu'il faut surtout faire attention aux différents sens du discours, pour bien ponctuer [...]. Cependant si on ne se proposoit que la distinction des sens partiels, sans égard aux besoins de la respiration; chacun placeroit les caractères distinctifs selon qu'il jugeroit convenable d'anatomiser plus ou moins les parties du discours: car la pensée étant essenciellement une \& indivisible, rien n'empêche qu'on ne regarde chaque proposition comme un tout également indivisible, quelque longue
} 
Un'ultima indicazione suggerisce l'appartenenza dell'interpunzione al complesso della grammatica generale - che contempla principi e norme ritenuti comuni a tutte le lingue, indagandone $\mathrm{i}$ fondamenti logici in relazione all'espressione analitica del pensiero - e giustifica in tal modo la presenza, nel lavoro, di un intero capitolo sull'argomento:

Un système de Ponctuation ne peut porter sur des fondements solides, s'ils ne sont pris dans les vûes de l'analyse de la pensée, qui est la Logique grammaticale. Mais alors ce système n'est pas plus propre à la langue françoise qu'à toute autre: c'est une partie de l'objet de la Grammaire générale [...]: c'est pour cela même que j'ai cru devoir en faire un chapitre exprès dans cet ouvrage ${ }^{39}$.

La finezza di tali ragionamenti, unita all'autorevolezza del grammatico e alla precoce attribuzione d'importanza al tema, rendono le proposte interpuntive formalizzate dal Beauzée degne di nota sino a Ottocento inoltrato ${ }^{40}$.

\section{ESEMPI MANZONIANI}

Nelle grammatiche italiane di secondo Ottocento, all'interpretazione pausativa e all'interpretazione morfo-sintattica del sistema interpuntivo si aggiunge una concezione diversa: l'interpretazione comunicativo-testuale. Se è vero che le prime due resistono soprattutto nei manuali di impronta puristica mentre l'ultima trova posto nei manuali toscano-manzoniani o in altri supporti che abbracciano un'idea moderna di lingua ${ }^{41}$, è anche lecito supporre che tra gli interlocutori a distanza dei grammatici francesi sia da comprendere Manzoni. Di seguito - lontana la presunzione di un lavoro esauriente intorno a un tema tanto articolato - qualche esempio significativo tratto dai Promessi Sposi $^{42}$, che parrebbe suggerire il ricorso a tale modello.

\subsection{La funzione esplicativa dei due punti.}

Un primo caso interessante è quello dei due punti. Tra le funzioni comunicative attualmente riconosciute al segno si annovera quella esplicativa, talora condivisa con il punto e virgola e il punto. A differenza di questi ultimi, però, il primo segno è semanticamente più ricco, poiché proietta sul testo un movimento compatto,

qu'elle puisse être d'ailleurs; \& les divisions que l'on y introduiroit tiendroient beaucoup de l'arbitraire. [...] Enfin si, dans la distinction des sens, on ne faisoit attention aux différences qui les subordonnent les uns aux autres, \& aux degrés de cette subordination; les parties subalternes du troisième ordre, par exemple, seroient séparées entre elles par des intervalles égaux à ceux qui distingueroient les parties du second ordre \& du premier: cette égalité des intervalles améneroit dans la prononciation une sorte d'équivoque, puisqu'elle présentorit comme parties également dépendantes d'un même tout, des sens réellement subordonnés les uns aux autres \& distingués par différents degrés d'affinité» (ivi : 578-579). I corsivi sono dell'autore. Non stupisce, dunque, che dopo Beauzée il sistema di puntazione si appoggi su tre principi: «Le premier est la sobriété [...]; cette sobriété s'appuie à son tour sur l'unité de la pensée totale [...]. Le troisième principe est la clarté de l'énonciation» (Lorenceau, 1998: 367).

${ }^{39}$ Ivi: 582.

40 A partire dal 1811 e per tutto il corso del secolo, per esempio, a esse si rifà esplicitamente la rinomatissima Grammaire des grammaires di Girault-Duvivier (per questo e altri riferimenti, cfr. Barsi, 2008b: 286).

${ }^{41}$ Cfr. anche Ferrari (2020: 3-5).

${ }^{42}$ L'edizione di riferimento per il testo dei Promessi Sposi è quella curata da Caretti (1971), che rende possibile e immediato il confronto tra le due edizioni del romanzo. Gli esempi sono tratti dai capitoli I, VIII, XIV, XXI, XXIII, sottoposti, in altra sede, a puntuale analisi. 
consentendo a chi legge di identificare il legame sussistente tra gli enunciati come elemento indispensabile per la corretta interpretazione della sequenza. In relazione al rapporto tra due punti e punto e virgola, inoltre, è utile precisare che la grammaticografia antica consentiva, quando non incoraggiava, il fluido interscambio dei due segni; nella punteggiatura moderna e contemporanea, invece, si assiste alla specializzazione dei due punti nei movimenti logici di tipo presentativo, mentre al punto e virgola sono associate funzioni perlopiù segmentanti-gerarchizzanti e isolanti.

Il ruolo chiarificatore dei due punti entra nella norma grammaticale italiana soltanto a partire dal Gherardini ${ }^{43}$; nel 1767, tuttavia, Beauzée già scriveva:

II. Règle. Si après une proposition qui a par elle-même un sens complet, \& dont le tour ne donne pas lieu d'attendre autre chose, on ajoûte une autre proposition qui serve d'explication ou d'extension à la première; il faut séparer l'une de l'autre par une Ponctuation plus forte d'un degré, que celle qui auroit distingué les parties de l'une ou de l'autre ${ }^{44}$.

Nel romanzo di Manzoni, la regola conosce una buona esemplificazione sin dalla Ventisettana:

(V) Era Perpetua, come ognuno se ne avvede, la serva di don Abbondio: serva affezionata e fedele, che sapeva ubbidire e comandare secondo l'occasione, tollerare a tempo i brontolamenti e le fantasticaggini del padrone, e fargli a tempo tollerare le sue, che divenivano di giorno in giorno più frequenti, dacchè ella aveva passata l'età sinodale dei quaranta, rimanendo celibe, per aver rifiutati tutti i partiti che le si erano offerti, com'ella diceva, o per non aver mai trovato un cane che la volesse, come dicevano le sue amiche. (Cap. I, S 65) Non c'era che dire: Agnese per non farle nascere qualche sospetto, aveva dovuto voltar con lei e andarle dietro, cercando però di soprattenerla ogni volta che la vedesse infervorata ben bene nel racconto di quei tali matrimonii andati a monte. (Cap. VIII, $\mathbb{\int} 47$ ) Però di questo muto soliloquio nulla trasparve sulla faccia dell'oste, la quale stava immobile come un ritratto: una faccia pienotta e lucente, con una barbetta folta, rossigna, e due occhietti chiari e fissi. (Cap. XIV, \23) Eran tutte sue, eran lui: l'orrore di questo pensiero, rinascente a ognuna di quell'immagini, attaccato a tutte, crebbe fino alla disperazione. (Cap. XXI, \50)

Notevole anche il seguente passaggio, in cui i due punti, collocati dopo l'onomatopea che riproduce i rintocchi della campana suonata dal sacrestano Ambrogio, danno avvio alla scena nella quale viene descritta la reazione del paese in fermento:

(V) Ton, ton, ton, ton: i contadini balzano a sedere sul letto; i garzoni sdraiati sul fenile, tendono l'orecchio e saltano in piedi. (Cap. VIII, \30)

Un impiego che tematizza, isolandolo a livello testuale e, fuor di testo, uditivo, l'elemento dominante dell'intero capitolo.

Nell'ambito della variantistica, sono diversi gli interventi che volgono in questa direzione e che sembrano determinare, in tal senso, la preferenza per i due punti in luogo del punto e virgola. Se ne vedano alcuni:

${ }^{43}$ Cfr. Gherardini (1843: 542). Non viene mai codificato, invece, per il punto e virgola.

${ }^{44}$ Beauzée (1767: 610). 
(V) Siete voi che lo volete. Ecco, io vi lascio il buon luogo; mi corco qui su la sponda; starò disagiata per voi.

(V) E sua signoria illustrissima, subito subito, a braccia aperte, caro amico, amico caro; stare a tutto quello che gli dice costui, come se lo avesse veduto far miracoli; e di lancio pigliare addirittura una risoluzione, darvi dentro colle mani e co' piedi, presto di qua, presto di là̀ a casa mia si chiama precipitazione.

(V) Basta; il cielo è in obbligo di aiutarmi, perchè non mi ci son messo io di mio capriccio.
(Q) Siete voi che lo volete. Ecco, io vi lascio il posto buono: mi metto sulla sponda; starò incomoda per voi. (Cap. XXI, \34)

(Q) E sua signoria illustrissima, subito subito, a braccia aperte, caro amico, amico caro; stare a tutto quel che gli dice costui, come se l'avesse visto far miracoli; e prendere addirittura una risoluzione, mettercisi dentro con le mani e co' piedi, presto di qua, presto di là: a casa mia si chiama precipitazione. (Cap. XXIII, \62)

(Q) Basta: il cielo è in obbligo d'aiutarmi, perchè non mi ci son messo io di mio capriccio. (Cap. XXIII, \65)

\subsection{La virgola del soggetto.}

Il caso della virgola tra soggetto e predicato viene teorizzato per la prima volta, in Italia, da Giuseppe Malagòli (1912), che scrive: «Non si mette mai la virgola dopo il soggetto, se questo è seguito immediatamente dal predicato», chiarendo poi, in una nota:

La cosa è diversa quando il soggetto venga dopo il predicato, oppure quando il soggetto sia complesso, cioè abbia de' complementi che stiano fra esso e il predicato in modo che possa esser dubbio se appartengono a questo o a quello: la virgola potrà giovare, allora, a togliere l'ambiguità, indicando nell'un caso la trasposizione, e nell'altro l'unione dei complementi al proprio sostantivo $[\ldots]^{45}$.

In territorio francese, invece, è Beauzée il primo a prescrivere l'inserimento di una virgola quando un enunciato superi, in ampiezza, l'intervallo di respirazione naturalmente sostenibile da chi legge. Lo scopo è quello di isolare e distinguere fra loro gli elementi che prendono parte all'enunciato stesso - tra i quali egli annovera, appunto, anche il soggetto logico - favorendone la comprensione.

Quanto ai Promessi Sposi, nella Ventisettana la tendenza a frapporre la virgola tra soggetto e predicato è perlopiù riscontrabile laddove il primo sia espanso, spesso tramite l'aggiunta di costituenti circostanziali. La presenza del segno trova, così, giustificazione nella volontà di sopperire alla distanza che si consuma tra i due elementi, ma soprattutto nell'esigenza di rimuovere eventuali oscurità di senso imputabili all'accumulo di determinazioni.

L'espansione può presentarsi in forma di subordinata esplicita (e in tal caso si realizza soprattutto mediante relativa attributiva o appositiva):

(V) Come il cane che scorta un gregge di porci, corre or qua or là a quei che si sbandano, ne addenta uno per un'orecchia e lo tira in ischiera, ne spinge un altro col muso, abbaia ad un altro che esce di fila in quel momento, cosí il pellegrino acciuffa uno di coloro che già toccava la soglia e lo strappa indietro, caccia indietro col bordone uno e un altro che v'eran già presso, grida agli altri che scorrazzano senza saper dove, tanto che li raccozzò tutti nel mezzo del cortiletto. (Cap. VIII, \46)

45 G. Malagòli (1912: 169). Poco oltre, l'autore propone due esempi di quanto affermato: il secondo è proprio da Manzoni. 
(V) Renzo che era il più in cervello di tutti, pensò che di qua o di là conveniva andar subito, prima che la gente accorresse, e che la più sicura era di fare ciò che Menico consigliava, anzi comandava colla forza d'uno spaventato. (Cap. VIII, \56)

\section{O implicita:}

(V) Quanto è tristo il passo di chi cresciuto tra voi, se ne allontana! (Cap. VIII, \94)

All'interno della Quarantana, il procedimento non viene sconfessato ${ }^{46}$, ma piuttosto precisato: l'aggiunta di numerose virgole si accorda, infatti, con la tendenza a regolarizzare quelle doppie, serrandovi all'interno gli elementi circostanziali ${ }^{47}$. Negli ultimi due passi elencati, per esempio, l'assenza della virgola d'apertura in costituenti che l'autore sente, sin dalla prima edizione, di dover chiudere (senza però racchiudere) viene colmata, e il testo presenta la seguente redazione finale:

(Q) Renzo $\mathbf{2}_{2}$ ch'era il più in sè di tutti ${ }_{2}$ pensò che, di qua o di là, conveniva andar subito, prima che la gente accorresse; e che la più sicura era di far ciò che Menico consigliava, anzi comandava, con la forza d'uno spaventato. (Cap. VIII, \56)

(Q) Quanto è tristo il passo di chi, cresciuto tra voi, se ne allontana! (Cap. VIII, \94)

Conosce regolarizzazione interna anche un passo come:

(V) Frattanto Tonio, e ad un suo cenno Gervaso, si posero in piedi dinanzi al tavolino in modo di togliere allo scrittore la vista della porta; e come per ozio andavano soffregando coi piedi il pavimento, per dar segno a quei di fuori che entrassero, e per confondere nello stesso tempo il romore delle loro pedate.
(Q) Frattanto Tonio e, a un suo cenno, Gervaso, si piantaron ritti davanti al tavolino, in maniera d'impedire allo scrivente la vista dell'uscio; e, come per ozio, andavano stropicciando, co' piedi, il pavimento, per dar segno a quei ch'erano fuori, d'entrare, e per confondere nello stesso tempo il rumore delle loro pedate. (Cap. VIII, \19)

in cui la virgola tra soggetto (Tonio e Gervaso) e predicato (rispettivamente si posero e si piantaron) permane, divenendo però più significativa. Nella prima versione, infatti, il secondo costituente soggettivale ha la forma di un'aggiunta; nell'ultima, invece, a essere racchiuso tra due virgole è il cenno di Tonio, mentre la virgola tra Gervaso e si piantaron è singola e ha la funzione di separare i due soggetti dal predicato corrispondente.

Nella Quarantana si riscontrano nuovi casi di costituenti nucleari soggetto isolati, in coordinazione asindetica:

(V) Anche le tenebre, anche il silenzio_gli facevano apprendere nella morte qualche cosa di più tristo, di spaurevole; gli pareva che non avrebbe esitato, se si trovasse al giorno chiaro, fuori, in faccia alla gente: gittarsi in un'acqua e sparire.
(Q) Anche le tenebre, anche il silenzio, gli facevan veder nella morte qualcosa di più tristo, di spaventevole; gli pareva che non avrebbe esitato, se fosse stato di giorno, all'aperto, in faccia alla gente: buttarsi in un fiume e sparire. (Cap. XXI, \52)

\footnotetext{
${ }^{46}$ Nessuna delle virgole precedentemente rilevate, infatti, subisce un'espunzione.

${ }^{47}$ Cfr. Ferrari (2020: 13).
} 
o sindetica ${ }^{48}$ :

(V) Si vede dunque chiaramente che il re_e quei che comandano_vorrebbero che i birbi fossero castigati; ma non se ne fa niente, perchè c'è una lega.
(Q) $\mathrm{Si}$ vede dunque chiaramente che il $\mathbf{r e}_{2} \mathrm{e}$ quelli che comandano, vorrebbero che i birboni fossero gastigati; ma non se ne fa nulla, perchè c'è una lega. (Cap. XIV, \$11)

Inoltre, l'impiego della virgola per isolare il soggetto si estende e diversifica, indice di una maggiore padronanza del testo e del segno. La situazione più semplice, soprattutto perché al di sopra dei più immediati sospetti di irregolarità, è quella in cui il soggetto isolato dalla virgola subisce una dislocazione che lo confina in fondo alla proposizione:

(V) Ma voglio che me lo paghi bene questo servizio_colui.
(Q) Ma voglio che me lo paghi bene questo servizio, colui. (Cap. XXI, \13)

In effetti, è ancora Beauzée a sottolineare come, in caso di iperbato di un singolo elemento o di una intera proposizione, l'inserimento di una o più virgole non sia soltanto auspicato, ma persino necessario ${ }^{49}$. Invece, in un passaggio come:

(V) Don Abbondio_intravvide, vide, si spaventò, si stupí, s'infuriò, pensò, prese una risoluzione: tutto questo nel tempo che Renzo mise a proferire le parole: «signor curato, in presenza di questi testimonii, quest'è mia moglie.»
(Q) Don Abbondio, vide confusamente, poi vide chiaro, si spaventò, si stupí, s’infuriò, pensò, prese una risoluzione: tutto questo nel tempo che Renzo mise a proferire le parole: «signor curato, in presenza di questi testimoni, quest'è mia moglie.» (Cap. VIII, \$21)

la virgola, contravvenendo a ogni prescrizione normativa, chiude un soggetto breve, puramente nominale, e a inizio frase: isola così il tema, enfatizzandolo. In tal modo, inoltre, il soggetto genera l'impressione di prendere parte alla serialità verbale, in rapporto di coordinazione asindetica, che da esso dipende, e contribuisce alla resa teatrale della scena. La sequenza è tratta infatti dal racconto dell'agguato che si svolge in casa del curato, e in particolare dal momento in cui Renzo e Lucia fanno la loro apparizione nella sala; Don Abbondio è l'ultimo personaggio - dopo Tonio, Gervaso e i due giovani - i cui pensieri e le cui azioni vengono presentati al lettore, prima che la situazione si risolva in un disordinato trambusto.

Nel passaggio seguente, la virgola addirittura chiude e tematizza un soggetto senza predicato $^{50}$ :

Menico_fuora, e a gambe per la contrada alla volta del campanile, dove a buon conto qualcheduno vi doveva essere.
Menico, via a gambe per la strada, alla volta del campanile, dove a buon conto qualcheduno ci doveva essere. (Cap. VIIII, \$ 44)

\footnotetext{
${ }^{48}$ Nella grammatica di Cappuccini e Morandi, si legge: «Quando però i due soggetti o i due complementi siano lunghi, il secondo si considera quasi sempre come un inciso, e si mette tra due virgole» (G. Cappuccini, L. Morandi, Grammatica italiana (regole ed esercizi), Torino, Paravia, 1894, citazione a p. 269). Per un caso affine già nella Ventisettana, cfr. Cap. VIII, $\int 19$.

${ }^{49}$ Cfr. Beauzée (1767: 596). In Italia, simile concessione è solo in Malagòli (cfr. supra).

${ }^{50}$ I primi a trattare del ruolo della virgola «nelle proposizioni ellittiche, nel luogo dov'è taciuto il verbo» sono di nuovo Cappuccini e Morandi (1894: 270). Il caso è reso ancor più significativo dal fatto che, nel passaggio dalla Ventisettana alla Quarantana, è attestata una generale tendenza alla correzione delle frasi ellittiche (cfr. Mencacci, 1995: 47-50).
} 
In tutti questi casi, le virgole aggiunte si oppongono alla sintassi: introducono, cioè, un «confine informativo laddove la sintassi tenderebbe a segnalare linearizzazione semantica all'interno della stessa Unità Informativa» ${ }^{51}$.

\subsection{Il corsivo di citazione.}

Uno tra i metodi di citazione in uso nel romanzo manzoniano consiste nell'impiego del corsivo $^{52}$. Nel capitolo I, per esempio, esso viene utilizzato per la menzione documentaria delle gride $^{53}$. Se ne legga un frammento:

(Q) Ma, nell'anno seguente, il 12 aprile, scorgendo il detto signore, che questa Città è tuttavia piena di detti bravi... tornati a vivere come prima vivevano, non punto mutato il costume loro, nè scemato il numero, dà fuori un'altra grida, ancor più vigorosa e notabile, nella quale, tra l'altre ordinazioni, prescrive: Che qualsivoglia persona, cosi di questa Città, come forestiera, che per due testimonj consterà esser tenuto, e comunemente riputato per bravo, et aver tal nome, ancorchè non si verifichi aver fatto delitto alcuno... per questa sola riputazione di bravo, senza altri indirj;, possa dai detti giudici e da ognuno di loro esser posto alla corda et al tormento, per processo informativo... et ancorchè non confessi delitto alcuno, tuttavia sia mandato alla galea, per detto triennio, per la sola opinione e nome di bravo, come di sopra. Tutto ciò, e il di più che si tralascia, perchè Sua Eccellenza è risoluta di voler essere obbedita da ognuno. (Cap. I, \16-17)

Tale impiego del carattere, non riscontrabile nelle grammatiche italiane setteottocentesche ${ }^{54}$, emerge al contrario molto presto in Francia, e in particolare tra le prescrizioni enciclopediche di Beauzée. Quest'ultimo si riferisce a una duplice opposizione da tenere presente quando si riportano, scrivendo, le parole altrui, vale a dire quella tra discours feint e discours écrit, prima, e quella tra caracter italique e caracter romain, poi:

Il faut remarquer que le discours direct que l'on rapporte, doit commencer par une lettre capitale, quoiqu'on ne mette pas un point à la fin de la phrase précédente. Si c'est un discours feint, comme ceux des exemples précédens, on a coûtume de le distinguer du reste par des guillemets: si c'est un discours écrit que l'on cite, il est assez ordinaire de le rapporter en un autre caractere que le reste du discours où celui-là est introduit, soit en opposant l'italique au romain, soit en opposant différens corps de caracteres, de l'une ou de l'autre de ces deux especes ${ }^{55}$.

La citazione tra virgolette, insomma, non avrebbe il medesimo significato di quella in corsivo, e la differenza starebbe nella distinzione tra discorso inventato dall'autore, e orale, e discorso documentato, scritto. In realtà, mentre le virgolette sembrano definire l'eterogeneità del testo scritto, demarcando esplicitamente un enunciato estraneo a quello

\footnotetext{
51 Ferrari et al. (2018: 53).

52 Pur distinguendosi dai segni interpuntivi prototipici, oggi il corsivo è annoverato tra i segni paragrafematici dell'italiano per la sua capacità di esprimere un valore distintivo e funzionale ai grafemi della lingua, intervenendo sulla loro forma. In questo senso è incluso nella presente trattazione. Per un'articolata disamina dell'argomento, cfr. Castellani (1985: 3-47).

${ }^{53} \mathrm{Ma}$ cfr. anche Cap. XIV, $\int 30$.

${ }^{54}$ In generale, esse non affrontano il tema del corsivo, a lungo considerato appannaggio del tipografo. Solo in Gherardini (1825: 135) si registra l'uso di «sottosegnare con linee o scrivere con diversa forma di carattere» le citazioni brevi d'autore.

${ }^{55}$ Beauzée (1973 [1751]: 22).
} 
principale, il corsivo favorisce, per converso, l'incorporazione della citazione stessa all'interno del testo ${ }^{56}$. A tale distinzione, il testo dei Promessi Sposi si attiene senza eccezioni.

\section{CONCLUSIONI}

Come si è potuto osservare, le opere dei principali grammatici illuministi riservano ampio spazio alla punteggiatura, consumando in tal modo un netto distacco rispetto alle sintetiche soluzioni adottate dai coevi grammatici italiani. Le differenze non sono solo quantitative, ma anche, e soprattutto, qualitative: le riflessioni di Claude Buffier, dell'abate Girard e di Nicolas Beauzée sono profonde e articolate, e oltrepassano presto, dapprima per cenni e poi in modo più consapevole e diffuso, la proposta binaria (divisa cioè tra pausazione e sintassi) fino a quel momento invalsa nell'interpretazione e conseguente codificazione della materia interpuntiva. Pur non pervenendo a soluzioni univoche o definitive, biasimano la scarsa attenzione sino a quel momento dedicata all'argomento e anticipano così un paradigma moderno di impiego della punteggiatura: quello comunicativo-testuale.

Tanto spazio e tanta modernità si leggono, in Italia, solo nelle grammatiche che appartengono alla seconda metà del secolo diciannovesimo, in particolare in quelle che accolgono e normalizzano un'idea non puristica di lingua o che, più specificamente, prendono a modello l'esempio offerto dal romanzo manzoniano. Sembra lecito supporre, dunque, che il cambiamento registrato dalle grammatiche italiane sia stato incoraggiato, se non addirittura veicolato, dalle scelte operate da Manzoni nei Promessi Sposi.

In effetti, una breve ricognizione del testo del romanzo, e degli interventi introdotti dall'autore nel passaggio dalla Ventisettana alla Quarantana, consente di notare quanto Manzoni sia in anticipo - di almeno mezzo secolo - rispetto alle prescrizioni delle grammatiche coeve. Naturalmente, la direzione delle scelte manzoniane non è omogenea né lineare, segno del fatto che probabilmente l'autore non aveva ancora assimilato definitivamente la concezione comunicativo-testuale della punteggiatura. Lo stimolo per un uso interpuntivo moderno può essere individuato nella sensibilità dell'autore nei confronti della funzionalità comunicativa delle strutture linguistiche; è tuttavia lecito supporre che possa provenire anche da un'attenta lettura e introiezione della lezione impartita dalle grammatiche del Settecento francese. Il che è probabile, almeno per due motivi: il primo, la forte influenza che la lingua francese esercitò sull'intera parabola biografica e letteraria di Manzoni; il secondo, la sua analitica conoscenza delle grammatiche illuministe, documentata dagli appunti inclusi nel trattato inedito Della lingua italiana.

Per questo motivo, si è scelto di proporre in conclusione una sintetica, e non esaustiva, casistica tratta dai Promessi Sposi, in cui sia possibile riconoscere delle convergenze tra la teorizzazione francese settecentesca e alcuni usi manzoniani, in seguito presi a modello dalla tradizione grammaticografica italiana. È il caso dei due punti in funzione esplicativa, oggetto, nella Quarantana, di un puntuale processo di regolarizzazione; dei complessi usi della virgola in relazione al soggetto; del corsivo di citazione, che ricalca in modo puntuale le distinzioni semantiche proposte in merito da Beauzée.

${ }^{56}$ Cfr. anche Persiani (1998: 148-150). 
(C) Italiano LinguaDue 2. 2021. A. Redaelli, Per un'interpretazione comunicativa della punteggiatura nelle grammatiche francesi del secolo XVIII: esempi di lettura manzoniana

\section{RIFERIMENTI BIBLIOGRAFICI}

Barsi M. (2008a), "La punteggiatura in Francia. Il Settecento", in Mortara Garavelli B. (a cura di), Storia della punteggiatura in Europa, Laterza, Bari-Roma, pp. 267-278.

Barsi M. (2008b), "La punteggiatura in Francia. L'Ottocento e il Novecento", in Mortara Garavelli B. (a cura di), Storia della punteggiatura in Europa, Laterza, Bari-Roma, pp. 278-293.

Beauzée N. (1767), Grammaire générale, ou exposition raisonnée des éléments nécessaires du langage, pour servir de fondement à l'étude de toutes les langues, Volume II, Barbou, Paris.

Beauzée N. (1973 [1751]), "Ponctuation”, in Diderot D., D'Alembert J.-B, Encyclopédie, ou Dictionnaire raisonné des sciences, des arts et des métiers, XIII, France expansion, Paris, pp. 15-24.

Buffier C. (1709), Grammaire françoise sur un plan nowveau, Nicolas Le Clerc, Paris.

Cappuccini G., Morandi L. (1894), Grammatica italiana (regole ed esercizi), Paravia, Torino.

Caretti L. (a cura di) (1971), Manzoni A., I Promessi Sposi nelle due edirioni del 1840 e del 1825 27 raffrontate tra loro; Storia della colonna infame, Volume II, Einaudi, Torino.

Castellani A. (1985), "Sulla formazione del sistema paragrafematico moderno", in Studi linguistici italiani, XXI, pp. 3-47.

Catach N. (1996), "Mise en place de la ponctuation moderne: XVIII ${ }^{\mathrm{e}}$-XX ${ }^{\mathrm{e}}$ siècle", in Ead., La ponctuation. (Histoire et système), Presses Universitaires de France, Paris, pp. 35-47.

Corticelli S. (1745), Regole ed osservazioni della lingua toscana ridotte a metodo per uso del seminario di Bologna, Lelio dalla Volpe, Bologna.

Du Marsais C. C. (1769), Logique et principes de grammaire, Barrois, Paris.

Ferrari A. (2017), "Il fondamento comunicativo della punteggiatura italiana contemporanea: il caso della virgola e del punto e virgola", in Studia de Cultura, 1, pp. 152-165.

Ferrari A. et al. (2018), La punteggiatura italiana contemporanea. Un'analisi comunicativo- testuale, Carocci, Roma.

Ferrari A. (2020), "Le virgole dei Promessi Sposi", in Ead. (a cura di), Capitoli di storia della punteggiatura italiana, Edizioni dell'Orso, Alessandria, pp. 3-17.

Fornaciari R. (1881), Sintassi italiana nell'uso moderno, Sansoni, Firenze.

Fornara S. (2001), "Introduzione", in Soave F., Gramatica ragionata della lingua italiana, a cura di Fornara S., Pescara, Libreria dell'Università, 2001, pp. 9-72.

Fornara S. (2008), "Il Settecento", in Mortara Garavelli B. (a cura di), Storia della punteggiatura in Europa, Laterza, Bari-Roma, pp. 159-177.

Gastaldi C. (1895), La punteggiatura nel periodare italiano e il suo uso, Isoardi, Cuneo.

Gherardini G. (1825), Introduzione alla grammatica italiana per uso della classe seconda delle scuole elementari, Dell'Imperiale Regia Stamperia, Milano.

Gherardini G. (1843), Lessigrafía italiana o sia maniera di scrivere le parole italiane proposta e messa a confronto con quella insegnata dal Vocabolario della Crusca, Bianchi di Giacomo, Milano.

Girard G. (1747), Les V rais principes de la langue française, on La parole réduite en méthode conformément aux lois de l'usage, en seize discours, Volume II, Le Breton, Paris.

Lorenceau A. (1998), "Sur la ponctuation au $18^{\text {ème }}$ siècle", in Dix-huitième Siècle, 10, pp. 363-378: https://www.persee.fr/doc/dhs_0070-6760_1978_num_10_1_1195.

Malagòli G. (1912), Ortoepia e ortografia italiana moderna, Hoepli, Milano.

Mencacci O. A. (1995), Le correzioni a «I Promessi Sposi»: alcune varianti sintattiche, Guerra, Perugia.

Persiani B. (1998), "L'interpunzione dell'Ortis e della prosa del secondo Settecento", in Studi di grammatica italiana, XVII, pp. 127-244. 
(C) Italiano LinguaDue 2. 2021. A. Redaelli, Per un'interpretazione comunicativa della punteggiatura nelle grammatiche francesi del secolo XVIII: esempi di lettura manzoniana

Restaut P. (1730), Principes généraux et raisonnés de la grammaire francoise. Par Demandes \& par Réponses, J. Desaint, Paris.

Rogacci B. (1711), Pratica, e compendiosa istruzzione a' principianti, circa l'uso emendato, \& elegante della Lingua Italiana: composta da un religioso della Compagnia di Gesù, Antonio de' Rossi, Roma.

Soave F. (1771), Gramatica ragionata della lingua italiana di Francesco Soave, Fratelli Faure, Parma.

Soresi P.- D. (1756), I Rudimenti della lingua italiana, Regio-ducal corte, Milano. 